Check for updates

Cite this: RSC Adv., 2017, 7, 35482

\title{
A novel compact cathode using sponge-like RANEY® nickel as the sulfur immobilizer for lithium-sulfur batteries
}

\author{
Xi Zhu, Jianhua Tian, Xiaoyan Liu, Wenlong Huang, Didi Luo, Zhaodong Wang \\ and Zhongqiang Shan (D) *
}

\begin{abstract}
A novel sulfur immobilizer named RANEY® nickel is introduced for lithium-sulfur batteries for the first time. The sulfur/RANEY $®$ nickel (S/RN) composite is prepared via an accessible solvent method. The key feature of RANEY® nickel is that it contains poriferous channels, which act as barriers against the loss of active materials due to physical and chemical adsorption during discharge/charge cycling. The chemical adsorption of RANEY® nickel for sulfur, verified using X-ray photoelectron spectroscopy (XPS), leads to the generation of $\mathrm{Ni}-\mathrm{S}$ chemical bonds. In addition, RANEY® nickel, possessing excellent conductivity, can improve the reaction kinetics of an electrode with a high rate capability. As a result, the S/RN cathode presents an initial discharge capacity of $1469 \mathrm{~mA} \mathrm{~h} \mathrm{~g}{ }^{-1}$, achieving a minimal capacity fade of $0.12 \%$ per cycle after 500 cycles at the rate of $0.5 \mathrm{C}$ with an excellent coulombic efficiency of around $99 \%$. Furthermore, the S/RN cathode is promising for use in compact batteries due to its high volumetric specific capacity.
\end{abstract}

Received 17th May 2017

Accepted 7th July 2017

DOI: $10.1039 / \mathrm{c} 7 \mathrm{ra05569b}$

rsc.li/rsc-advances adsorption. ${ }^{16}$ Tao et al. reported a strategy of decorating pristine S with Pt nanostructures, and the Pt@S cathode shows only 15\% capacity fading after 80 cycles at $0.1 \mathrm{C} .{ }^{17}$ Nevertheless, most such embellished carbon frameworks are usually very light, with low densities of $0.3-0.6 \mathrm{~g} \mathrm{~cm}^{-3}$, leading to an inferior volumetric specific capacity of the electrode. The volumetric specific capacity of an electrode is important in many fields, in particular for applications in mini volume products, ${ }^{\mathbf{1 8 , 1 9}}$ and therefore, some studies were conducted to augment the volumetric specific capacity of electrodes. ${ }^{20-23}$ Zhang et al. synthesized highdensity graphene/sulfur electrodes with a high volumetric specific capacity for Li-S batteries. ${ }^{20}$ Wang et al. chemically bonded PS to both doped PCH and a 3D doped CP current collector to obtain a high sulfur loading cathode of $9.0 \mathrm{mg} \mathrm{cm}^{-2}$ with a slow capacity fading rate of $0.074 \%$ per cycle for 300 cycles. $^{23}$

Herein, we propose a new immobilizer material named RANEY® nickel to prepare sulfur/RANEY® nickel (S/RN) cathodes for lithium-sulfur batteries. RANEY® nickel is a metal sponge-like porous material with multiple integrated advantages. $^{24,25}$ To begin with, sulfur is homogeneously deposited on the microporous and mesoporous channels of RANEY® nickel, and the multilayered barriers of RANEY® nickel can restrict sulfur and polysulfides in the channels by physical adsorption and chemical bonding. ${ }^{26}$ Moreover, RANEY® nickel has good conductivity $\left(1.4 \times 10^{5} \mathrm{~S} \mathrm{~cm}^{-1}\right)$, which can improve the reaction kinetics of the sulfur electrode. At last, RANEY® nickel can enhance the volumetric specific capacity of the electrode when the amount of loaded sulfur is consistent with the sulfur/carbon 
(S/C) composite. The S/RN composite was prepared via an accessible method using tetrahydrofuran as solvent and vacuum evaporation induced drying. As anticipated, the S/RN cathode has an initial discharge capacity of $1469 \mathrm{~mA} \mathrm{~h} \mathrm{~g}^{-1}$, and achieves a minimal capacity loss of $0.12 \%$ per cycle after 500 cycles with a coulombic efficiency of $99 \%$.

\section{Experimental}

\section{Pretreatment processing of RANEY® nickel}

Initially, stoichiometric amounts of pristine RANEY® nickel (AR, Aladdin, China) and $20 \mathrm{wt} \% \mathrm{NaOH}$ solution (AR, Aladdin, China) were mixed using mechanical stirring at $50{ }^{\circ} \mathrm{C}$ for $24 \mathrm{~h}$. The product was subsequently washed several times with distilled water until the $\mathrm{pH}$ value was 7 , then the black product dispersed into distilled water was sonicated for $20 \mathrm{~min}$. Finally, the product was filtered and dried at $30{ }^{\circ} \mathrm{C}$ for $24 \mathrm{~h}$ under vacuum. The as-obtained product was denoted as RN.

\section{Preparation of S/RN and S/C composites}

The sulfur/RANEY® nickel (S/RN) composite was prepared via the following processes. A stoichiometric amount of sublimed sulfur (AR, Aladdin, China) was mixed with tetrahydrofuran (AR, Aladdin, China), and the solution was stirred for around $30 \mathrm{~min}$ until the solid sublimed sulfur was dissolved completely. Then a stoichiometric amount of RANEY® nickel powder was added to the solution, followed by sonication for $1 \mathrm{~h}$. The solvent was then evaporated at $30{ }^{\circ} \mathrm{C}$ under vacuum with a rotary evaporator. Afterwards, the damp-dry and slimy product was dried at $50{ }^{\circ} \mathrm{C}$ under vacuum for $5 \mathrm{~h}$. The product was denoted as $\mathrm{S} / \mathrm{RN}-X$ where $X$ represents the mass percentage of sulfur in the composite.

The sulfur/carbon (S/C) composite was prepared via the same procedures as the S/RN composite except for the matrix material, which was BP2000 (Black Pearls 2000, Cabot Corporation, America). This product was denoted as $\mathrm{S} / \mathrm{C}-X$ where $X$ presents the mass percentage of sulfur in the composite.

\section{Physical characterization}

The morphology and structure of pristine materials and composites were characterized using a field emission scanning electron microscope (FESEM, HITACHI, S-4800) equipped with an energy dispersive spectrometer (EDS). X-ray diffraction (XRD) (Bruker D8 Focus, $\mathrm{Cu} \mathrm{K} \alpha$ X-ray source) patterns of pristine materials and composites were measured in the $2 \theta$ range from $10-80^{\circ}$. Thermogravimetric analysis (TGA) was performed using a TA SDT Q600 in an $\mathrm{N}_{2}$ atmosphere from 40 to $700{ }^{\circ} \mathrm{C}$ at a heating rate of $10{ }^{\circ} \mathrm{C} \mathrm{min}^{-1}$. X-ray photoelectron spectroscopy (XPS) was used to analyze the surface elemental composition of hybrids. The Brunauer Emmett Teller (BET) method was used to measure the specific surface area and pore volume of RANEY ${ }^{\circledR}$ nickel and BP2000.

\section{Electrochemical characterization}

Electrochemical characterization of the composites was carried out by assembling a CR2023 coin cell in an argon-filled glove box (DELLIX, Chengtu, China). The electrode consisted of 80 wt $\% \mathrm{~S} / \mathrm{RN}$ or S/C composite, $10 \mathrm{wt} \%$ acetylene black, and 10 wt $\%$ polyvinylidene fluoride (PVDF) binder. The mixture dispersed in $N$-methylpyrrolidone (NMP) was spread on a carbon-coated aluminum foil and dried in a vacuum oven at $60{ }^{\circ} \mathrm{C}$ for around $10 \mathrm{~h}$. The obtained film was cut into discs with diameters of $14 \mathrm{~mm}$ using a punching method. The mass of sulfur loaded onto each round cathode was $0.8-1.1 \mathrm{mg} \mathrm{cm}^{-2}$. The electrolyte consisted of $1.0 \mathrm{M}$ lithium bis(trifluoromethane) sulfonamide (LiTFSI) in 1,2-dimethoxyethane (DME) and 1,3dioxolane with a volume ratio of $1: 1$ containing $1 \mathrm{wt} \% \mathrm{LiNO}_{3}$. The discharge/charge behavior of the cathode was tested on a Land CT2001A battery testing system with a voltage range of $1.7-2.8 \mathrm{~V}$ at $25{ }^{\circ} \mathrm{C}$. Cyclic voltammograms were collected between 1.5 and $3.0 \mathrm{~V}$ versus $\mathrm{Li}^{+} / \mathrm{Li}$ with a scan rate of $0.1 \mathrm{mV} \mathrm{s}^{-1}$ on the CHI66E electrochemical measurement system. Electrochemical impedance spectroscopy (EIS) was performed in a frequency range between $1 \mathrm{MHz}$ and $10 \mathrm{mHz}$ on an IM6e at open circuit voltage $(\sim 2.8 \mathrm{~V})$.

\section{Results and discussion}

The morphology and structure of RANEY® nickel and the S/RN composite were characterized using a scanning electron microscope (SEM). As demonstrated in Fig. 1a, the SEM image shows the sponge-like poriferous structure of RANEY® nickel. The framework can furnish dwelling sites for sulfur grains. As shown in Fig. 1b, the sulfur grains, after evaporating the solvent, can be easily captured by the sponge-like matrix. Upon careful observation, the size distribution of the sulfur grains is between $20-25 \mathrm{~nm}$, which is smaller than that of the bulk RANEY $®$ nickel. The reason for this may be that the sponge-like structure after activation exposes more surfaces, accordingly offering more deposition sites compared to pristine RANEY® nickel, and this facilitates the nucleation of sulfur from the solvent. The good dispersion of sulfur grains in the activated RANEY® nickel was further confirmed using EDS. As revealed by Fig. 2, the elemental content of nickel, aluminum and sulfur can be well distinguished. More importantly, the sulfur grains are well-distributed throughout the activated RANEY® nickel, which means that the strategy of using vacuum evaporationinduced solution to obtain the S/RN composite is very effective. Additionally, the uniform dispersion of sulfur has the great advantage of decreasing the adverse effects due to the intrinsic insulating character.

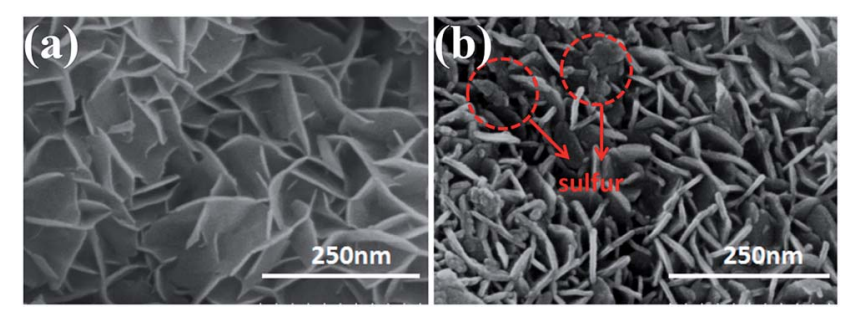

Fig. 1 (a) An SEM image of RN and (b) an SEM image of the S/RN composite. 


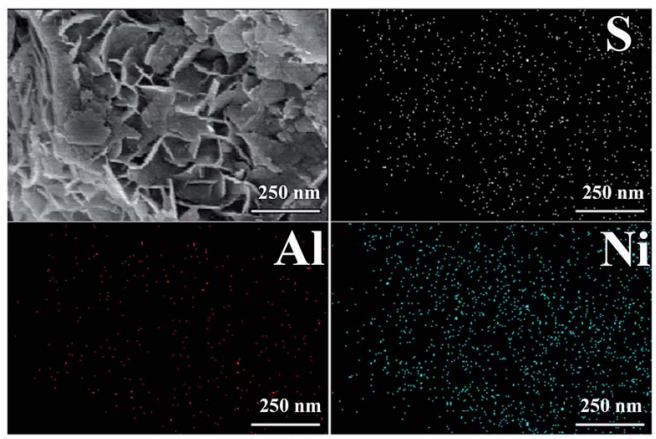

Fig. 2 EDS mapping images of the S/RN composite.

$\mathrm{N}_{2}$ adsorption-desorption isotherms were conducted in order to investigate the pore structure of BP2000 and RANEY® nickel, as demonstrated in Fig. 3. BP2000 possesses a specific surface area of $1258.2 \mathrm{~m}^{2} \mathrm{~g}^{-1}$ and a total pore volume of 0.90 $\mathrm{cm}^{3} \mathrm{~g}^{-1}$ (Fig. 3a and c). The Brunauer-Emmett-Teller (BET) method was used to calculate the specific surface area of RANEY® nickel, which was $153.7 \mathrm{~m}^{2} \mathrm{~g}^{-1}$. The pore volume is determined to be $0.16 \mathrm{~cm}^{3} \mathrm{~g}^{-1}$ and the micropore volume is 0.06 $\mathrm{cm}^{3} \mathrm{~g}^{-1}$. The RANEY® nickel framework displays a type I and IV isotherm curve following IUPAC classification, ${ }^{\mathbf{1 1}}$ confirming the typical microporous and mesoporous structure. The approximately vertical rise in the low-pressure range $\left(P / P_{0} \sim 0\right)$ indicates the presence of micropores. The typical hysteresis loop suggests the existence of mesopores at $P / P_{0}$ of $0.4-0.99$. The rise of the adsorption isotherms at $P / P_{0}$ near 1.0 is relative to the macropores. The macropores may be due to the dissolution of major aluminum in the sodium hydroxide solution. The pore size distribution of RANEY® nickel was estimated via the BarrettJoyner-Halenda (BJH) method, and this revealed that the pore size distribution was mostly centered at 12.5-50 nm with an average pore size of $18 \mathrm{~nm}$. According to previous literature, ${ }^{\mathbf{1 , 2 7}, 28}$ $\mathrm{S}_{8}$ molecules $(\sim 0.7 \mathrm{~nm})$ cannot be embedded into micropores smaller than $0.69 \mathrm{~nm}$, while the smaller molecules like $\mathrm{S}_{2-4}$ can easily be loaded into the pores. Hence, sulfur grains are easily (a)

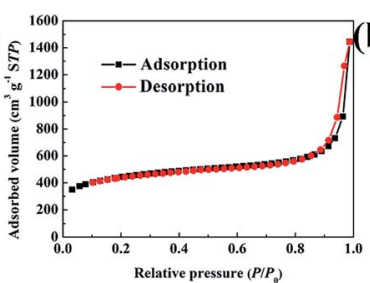

(c)

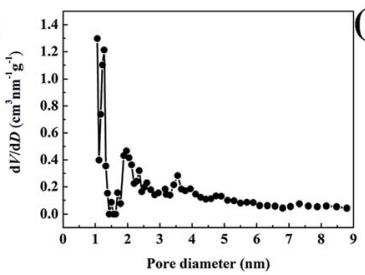

(d)

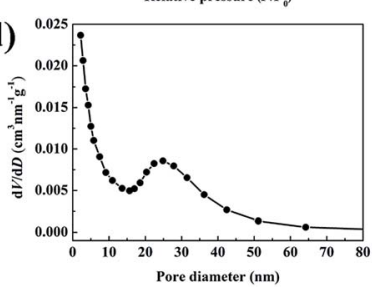

Fig. 3 BET nitrogen adsorption-desorption isotherms of (a) BP2000 and (b) RN and the pore size distribution plots of (c) BP2000 and (d) $\mathrm{RN}$. impregnated into the micropores and mesopores of RANEY ${ }^{\circledR}$ nickel with close contact.

To carry out the structural characterization and phase composition analysis of the S/RN and S/C composites, X-ray diffraction (XRD) patterns were collected. Fig. 4a shows the XRD patterns of sulfur, carbon, RANEY® nickel, and the S/RN and S/C composites. The sharp diffraction peaks in the XRD spectrum of elemental sulfur indicate the crystalline state of sulfur. ${ }^{29}$ The XRD spectrum of the S/RN composite displays a broad sharp peak at $2 \theta=23-29^{\circ}$, illustrating the crystalline state of sulfur in RANEY® nickel. The relatively lower peak intensity of sulfur in the S/RN composite as compared with that of elemental sulfur reflects that some sulfur grains are encapsulated within the micropores and mesopores of the RANEY® nickel, which reduces the crystal size of sulfur to the nanoscale. ${ }^{30}$ The XRD spectrum of RANEY® nickel shows an intense peak at $45^{\circ}$ and weak peaks at $53^{\circ}$ and $77^{\circ}$, representing elemental nickel. The two weak diffraction peaks at $38^{\circ}$ and $63^{\circ}$ correspond to the $\mathrm{Ni}_{x} \mathrm{Al}_{y} \mathrm{O}_{z}$ compound derived from the generation processes of the RANEY® nickel alloy. After loading with sulfur, some additional peaks appear at 30,37 , and $55^{\circ}$ in the XRD spectrum of the S/RN composite, which can be attributed to the (100), (101), and (110) planes of the hexagonal lattice of the NiS compound, respectively. The NiS peak indicates that the processes of immobilizing sulfur generate chemical bonds between sulfur and nickel. In comparison, the sharp sulfur
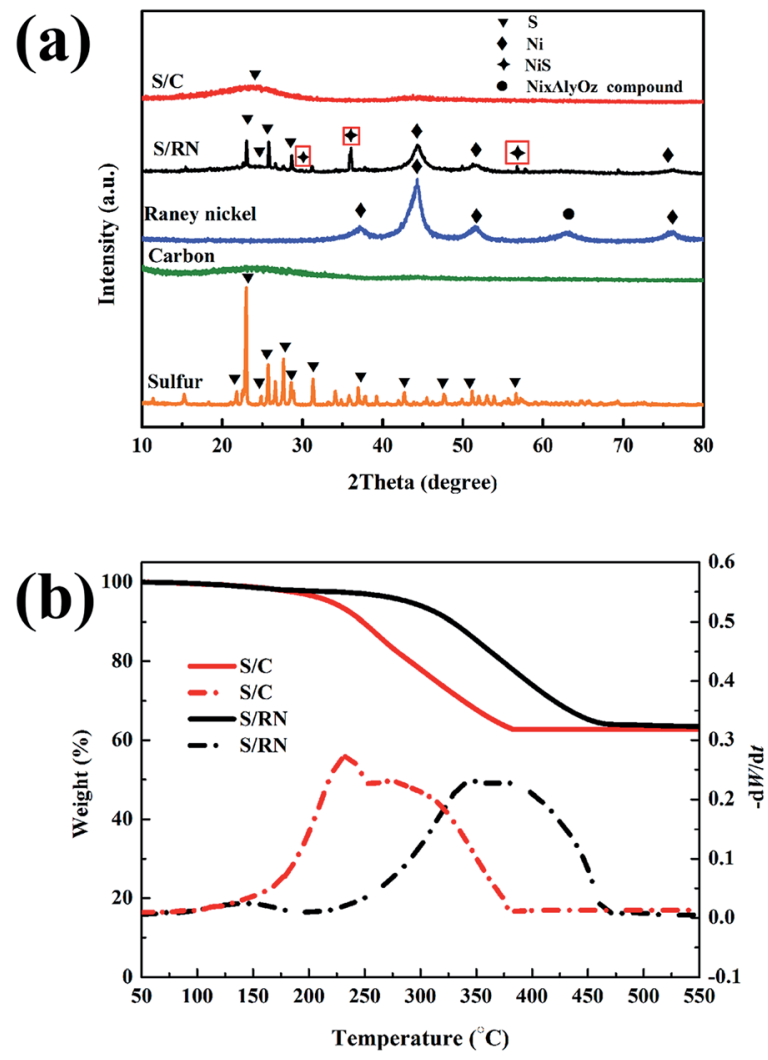

Fig. 4 (a) XRD patterns of sulfur, carbon, RANEY® nickel, and the S/RN and S/C composites; (b) TGA curves of the S/RN and S/C composites recorded in a $\mathrm{N}_{2}$ atmosphere. 
peaks disappear completely in the S/C composite, indicating that sulfur is highly dispersed inside the holes of carbon. Meanwhile, no new peaks appear in the XRD spectrum of the $\mathrm{S} /$ $\mathrm{C}$ composite, which means that there is no chemical reaction between sulfur and carbon.

In order to clarify the sulfur content and loading patterns of the $\mathrm{S} / \mathrm{RN}$ and $\mathrm{S} / \mathrm{C}$ composites, thermogravimetric analysis (TGA) was carried out. As shown in Fig. 4b, the final weight loss of the curves indicates that the sulfur loading content in the $\mathrm{S} / \mathrm{RN}$ and $\mathrm{S} / \mathrm{C}$ composites is $36.6 \mathrm{wt} \%$ and $37.3 \mathrm{wt} \%$, respectively. In detail, an initial weight loss of the S/RN composite appears at $225{ }^{\circ} \mathrm{C}$, and maximum weight loss is reached at $460{ }^{\circ} \mathrm{C}$. During the whole heating processes, the temperature of the highest sulfur loss rate in the $\mathrm{S} / \mathrm{RN}$ composite is between $350{ }^{\circ} \mathrm{C}$ and $400{ }^{\circ} \mathrm{C}$. Meanwhile, the $\mathrm{S} / \mathrm{C}$ composite demonstrates a temperature range of sulfur loss from $150{ }^{\circ} \mathrm{C}$ to $375^{\circ} \mathrm{C}$ and higher loss rate between $225{ }^{\circ} \mathrm{C}$ and $300{ }^{\circ} \mathrm{C}$, which are both lower than those of the $\mathrm{S} / \mathrm{RN}$ composite. The lower sulfur loss rate of the $\mathrm{S} / \mathrm{RN}$ composite means that higher energy is needed in the heating process. Therefore, after evaporation, sulfur is immobilized tightly in the deep channels by the interaction of sulfur and the RANEY® nickel immobilizer discussed below, exhibiting a hysteresis of sulfur loss in the TGA curve. ${ }^{31}$

Cyclic voltammograms (CVs) of the S/RN and S/C cathodes in the first three cycles were recorded. As shown in Fig. 5a and b, the $\mathrm{CV}$ curves of the $\mathrm{S} / \mathrm{RN}$ cathode present two distinct reduction peaks at about $2.3 \mathrm{~V}$ and $2.0 \mathrm{~V}$ in the cathodic scan, matching with the two reduction processes of transforming sulfur to soluble polysulfides $\left(\mathrm{Li}_{2} \mathrm{~S}_{n}, 2<n \leq 8\right)$ and reacting the soluble polysulfides to insoluble $\mathrm{Li}_{2} \mathrm{~S}_{2}$ and $\mathrm{Li}_{2} \mathrm{~S}$, respectively. ${ }^{32}$ Meanwhile, the broad oxidation peak at approximately $2.40 \mathrm{~V}$ in the anodic scan corresponds to the transformation from $\mathrm{Li}_{2} \mathrm{~S}_{2}$ and $\mathrm{Li}_{2} \mathrm{~S}$ to $\mathrm{Li}_{2} \mathrm{~S}_{n}(2<n \leq 8)$ and eventually to $\mathrm{S}_{8}$ during charging processes. ${ }^{27}$ The $\mathrm{CV}$ curve of the first cycle is different from the subsequent ones in the $\mathrm{S} / \mathrm{RN}$ and $\mathrm{S} / \mathrm{C}$ cathodes, implying a short activated stage of the cathodes. In addition, the second and third CV curves of the S/RN cathode overlap very well, indicating high electrochemical stability. It was most likely that polysulfides are prohibited effectively in the channels of the RANEY ${ }^{\circledR}$ nickel, and thus the shuttle effect is alleviated. ${ }^{14}$ Comparing the reduction peaks of the $\mathrm{S} / \mathrm{RN}$ cathode with that of $\mathrm{S} / \mathrm{C}$ cathode at around 2.3 and $2.0 \mathrm{~V}$ in the first cycle, the precise reduction peaks of the $\mathrm{S} / \mathrm{RN}(2.33$ and $2.04 \mathrm{~V})$ cathode are a bit larger than those of the S/C cathode (2.32 and $2.00 \mathrm{~V})$. Additionally, the oxidation peak of the $\mathrm{S} / \mathrm{RN}$ cathode $(2.39 \mathrm{~V})$ is lower that of the $\mathrm{S} / \mathrm{C}$ cathode $(2.43 \mathrm{~V})$. These data demonstrate that the reversibility of the $\mathrm{S} / \mathrm{RN}$ cathode is superior to that of $\mathrm{S} / \mathrm{C}$ cathode. Meanwhile, the reduction and oxidation peak areas of the S/RN cathode are larger than those of the S/C cathode, which indicates that the $\mathrm{S} / \mathrm{RN}$ cathode can provide more capacity.

Fig. 6a shows the cycling performances of the S/RN and S/C cathodes. The discharge capacities are calculated based on the mass of sulfur. The $\mathrm{S} / \mathrm{RN}$ cathode presents an initial discharge capacity of $1469 \mathrm{~mA} \mathrm{~h} \mathrm{~g}^{-1}$ at the rate of $0.5 \mathrm{C}$. With activation and stabilization, the discharge capacity is maintained at 758 $\mathrm{mA} \mathrm{h} \mathrm{g}^{-1}$ after 200 cycles (Fig. 6a). The S/RN cathode is further
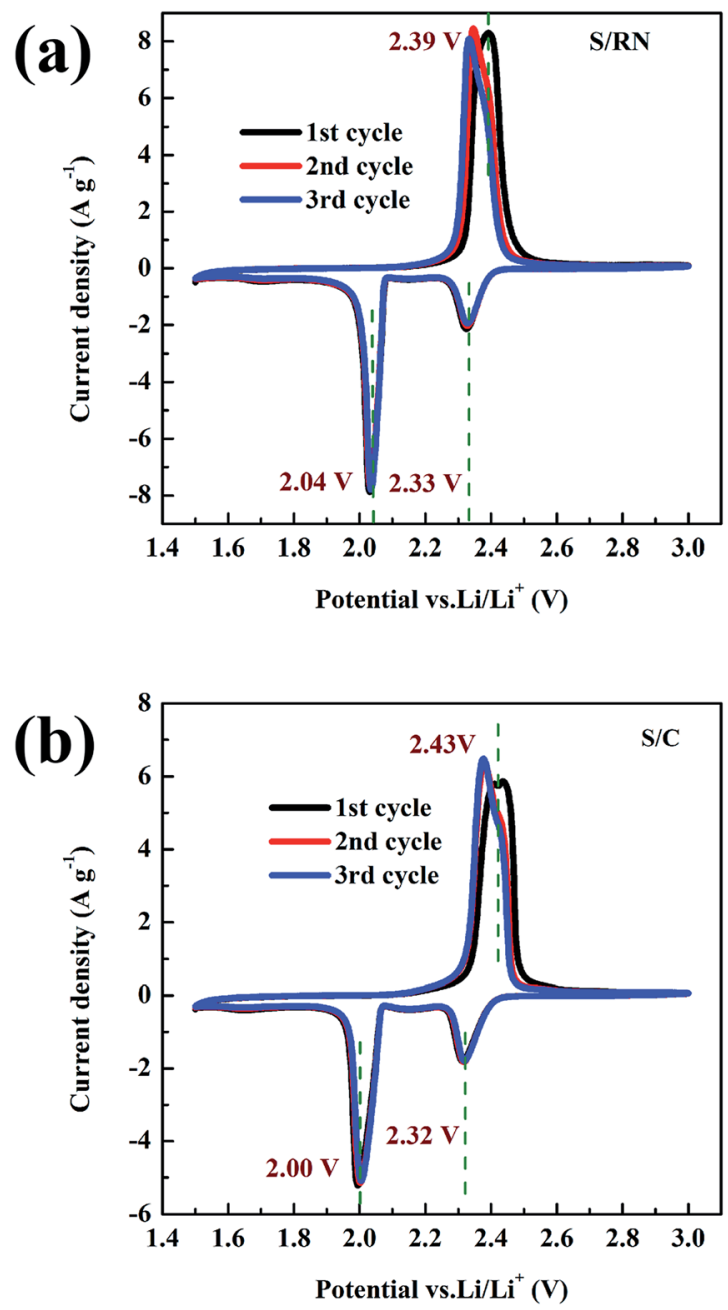

Fig. 5 The initial three CV curves of (a) the S/RN cathode and (b) the S/ C cathode.

subjected to long cycling of 500 cycles with a minimal capacity fade of $0.12 \%$ per cycle at the rate of $0.5 \mathrm{C}$ (Fig. $6 \mathrm{c}$ ). Nevertheless, the conventional $\mathrm{S} / \mathrm{C}$ cathode performs at a capacity retention of only $36 \%$ after 200 cycles with a rapid capacity fade of around $0.32 \%$ per cycle (Fig. 6a). More importantly, the S/RN cathode shows excellent stability in the coulombic efficiency ( 99\%) upon cycling, which is higher than that of the S/C cathode (93\%). The good discharge capacity retention of the S/RN cathode may be due to the physical and chemical adsorption of polysulfides in the micropores and mesopores of the RANEY® nickel. Furthermore, according to previous literature, metal oxides have the ability to chemically adsorb polysulfides, so a small amount of $\mathrm{Ni}_{x} \mathrm{Al}_{y} \mathrm{O}_{z}$ compounds in the RANEY® nickel carrier may act as adsorbers for polysulfides and inhibit the polysulfide shuttle effect.

Fig. 6b displays the volumetric and gravimetric specific capacities of the S/RN and S/C cathodes, which are calculated based on the whole volume and mass of the electrode. The volumetric specific capacity of the $\mathrm{S} / \mathrm{RN}$ cathode is $287 \mathrm{~mA} \mathrm{~h}$ $\mathrm{cm}^{-3}$ in the initial cycle and $148 \mathrm{~mA} \mathrm{~h} \mathrm{~cm} \mathrm{~cm}^{-3}$ after 200 cycles at the rate of $0.5 \mathrm{C}$, which is higher than that of the $\mathrm{S} / \mathrm{C}$ cathode 
(a)
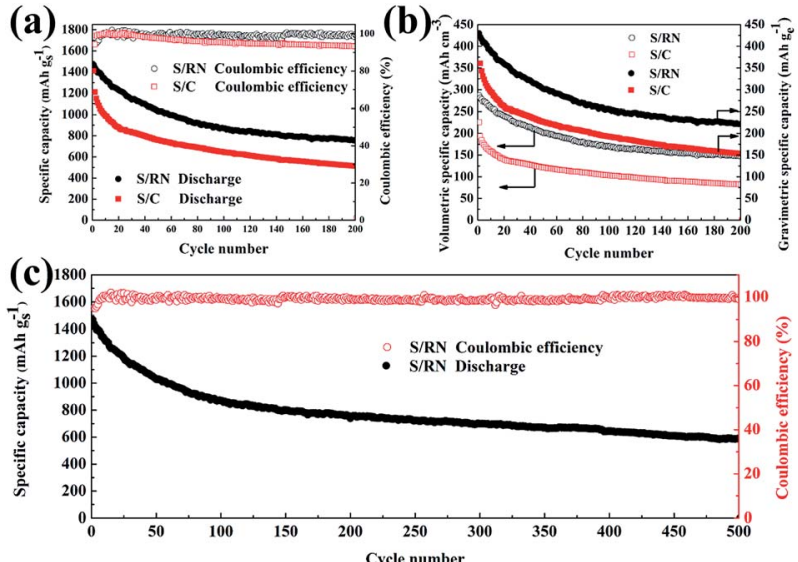

(d)

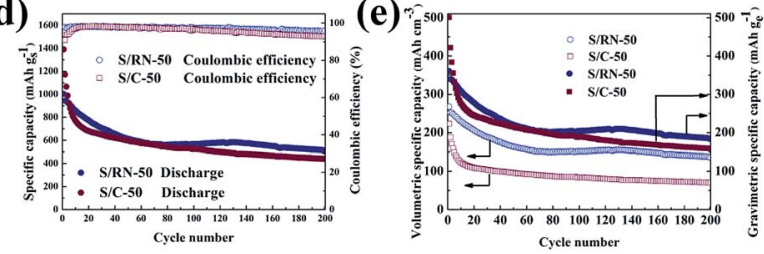

Fig. 6 (a) Cycling performances of the S/RN and S/C cathodes based on the mass of sulfur at the rate of $0.5 \mathrm{C}$; (b) volumetric and gravimetric specific capacities of the S/RN and S/C cathodes based on the electrode; (c) long-term cycling performance of the S/RN cathode based on the mass of sulfur at the rate of $0.5 \mathrm{C}$; (d) cycling performances of the S/RN-50 and S/C-50 cathodes based on the mass of sulfur at the rate of $0.5 \mathrm{C}$; (e) volumetric and gravimetric specific capacities of the $\mathrm{S} /$ RN-50 and S/C-50 cathodes based on the electrode.

either in the initial cycle $\left(226 \mathrm{~mA} \mathrm{~h} \mathrm{~cm}^{-3}\right)$ or after 200 cycles $(82$ $\mathrm{mA} \mathrm{h} \mathrm{cm}{ }^{-3}$ ). The obtained volumetric specific capacity after 200 cycles of the S/RN cathode is $80 \%$ higher than that of the S/C cathode. The high volumetric specific capacity cathode is promising for applications in compact Li-S batteries. ${ }^{19,20}$ Meanwhile, the S/RN cathode shows a gravimetric specific capacity of $430 \mathrm{~mA} \mathrm{~h} \mathrm{~g}^{-1}$ in the initial cycle and retains 222 $\mathrm{mA} \mathrm{h} \mathrm{g}{ }^{-1}$ in the $200^{\text {th }}$ cycle, which is $45 \%$ higher than that of the $\mathrm{S} / \mathrm{C}$ cathode $\left(153 \mathrm{~mA} \mathrm{~h} \mathrm{~g}^{-1}\right)$ in the $200^{\text {th }}$ cycle.

Sulfur mass loading in the composites has a dramatic effect on the cathode, and thus the cycling behaviors of the S/RN-50 cathode are conducted at the rate of $0.5 \mathrm{C}$, as shown in Fig. $6 \mathrm{~d}$ and e. The initial discharge capacities of the S/RN-50 and S/C-50 cathodes are 1002 and $1391 \mathrm{~mA} \mathrm{~h} \mathrm{~g}^{-1}$, respectively. After 200 cycles, the reversible capacities of the S/RN-50 and S/C-50 cathodes are 510 and $440 \mathrm{~mA} \mathrm{~h} \mathrm{~g}{ }^{-1}$. The coulombic efficiencies of S/RN-50 and S/C-50 cathodes are about $96 \%$ and $92 \%$, respectively. Hence, the cycling performance of the S/RN-50 cathode is still superior to that of the S/C-50 cathode, although the specific capacity decreases with the increase of sulfur mass loading. As displayed in Fig. 6e, the S/RN-50 and S/ C-50 cathodes deliver initial volumetric specific capacities of 268 and $223 \mathrm{~mA} \mathrm{~h} \mathrm{~cm}^{-3}$, respectively. The maintained volumetric specific capacity of S/RN-50 and S/C-50 cathode after 200 cycles are 136 and $71 \mathrm{~mA} \mathrm{~h} \mathrm{~cm}^{-3}$, respectively. So the obtained volumetric specific capacity after 200 cycles of S/RN-50 cathode has $92 \%$ higher than that of S/C-50 cathode. Comparing the gravimetric specific capacities of the S/RN-50 and S/C-50 cathodes, the S/RN-50 cathode still performs at a $16 \%$ higher capacity than the S/C-50 cathode in the $200^{\text {th }}$ cycle.

Fig. 7a illustrates the rate capabilities of the S/RN and S/C cathodes at different rates. As demonstrated in Fig. 7a, the S/ $\mathrm{RN}$ cathode delivers a discharge capacity of $1533 \mathrm{~mA} \mathrm{~h} \mathrm{~g}^{-1}$ in the first cycle at the rate of $0.1 \mathrm{C}$ and retains a discharge capacity of $1169 \mathrm{~mA} \mathrm{~h} \mathrm{~g}^{-1}$ after 10 cycles. Subsequently, the discharge capacities are around 1095, 983, 883 and $804 \mathrm{~mA} \mathrm{~h} \mathrm{~g}^{-1}$ in the first discharge curves at the high rates of $0.2,0.5,1$, and $2 \mathrm{C}$, respectively. As the rate was restored to $0.1 \mathrm{C}$, a reversible discharge capacity of $1035 \mathrm{~mA} \mathrm{~h} \mathrm{~g}{ }^{-1}$ is achieved, showing the good stability of the electrode material after high current density tests. In comparison, the $\mathrm{S} / \mathrm{C}$ cathode exhibits relatively lower discharge capacities of 1486, 819, 658, 554, and $470 \mathrm{~mA} \mathrm{~h}$ $\mathrm{g}^{-1}$ in the first discharge curves at the rates of $0.1,0.2,0.5,1$, and $2 \mathrm{C}$, respectively. The good rate discharge capacity of the $\mathrm{S} /$ RN cathode results from the decent electrical conductivity of RANEY® nickel.

Fig. $7 \mathrm{~b}$ and c display the voltage profiles of the S/RN and S/C cathodes. There are two discharge plateaus of the S/RN and S/C cathodes, in agreement with the reduction peaks illustrated in the $\mathrm{CV}$ curves. The overlapping plateaus of the S/RN and S/C cathodes also correspond to the oxidation peaks depicted in the $\mathrm{CV}$ curves. In addition, the S/RN cathode displays a smaller change in discharge plateaus compared with the S/C cathode on account of the strong chemical immobilization and controlled deposition of the sulfur/polysulfides in the channels of RANEY® nickel. ${ }^{33}$ The reduction voltages of the S/RN cathode are higher than those of the S/C cathode, showing a weak polarization effect resulting in promoted kinetics from the RANEY® nickel. The $\Delta E$ of the $\mathrm{S} / \mathrm{RN}$ cathode (between the charge plateau and the lower discharge plateau) is $0.35 \mathrm{~V}$ which is lower than that of the $\mathrm{S} / \mathrm{C}$ cathode $(0.43 \mathrm{~V})$, further implying a decrease in polarization and accelerated kinetics for the polysulfide redox induced by RANEY ${ }^{\circledR}$ nickel. ${ }^{34}$
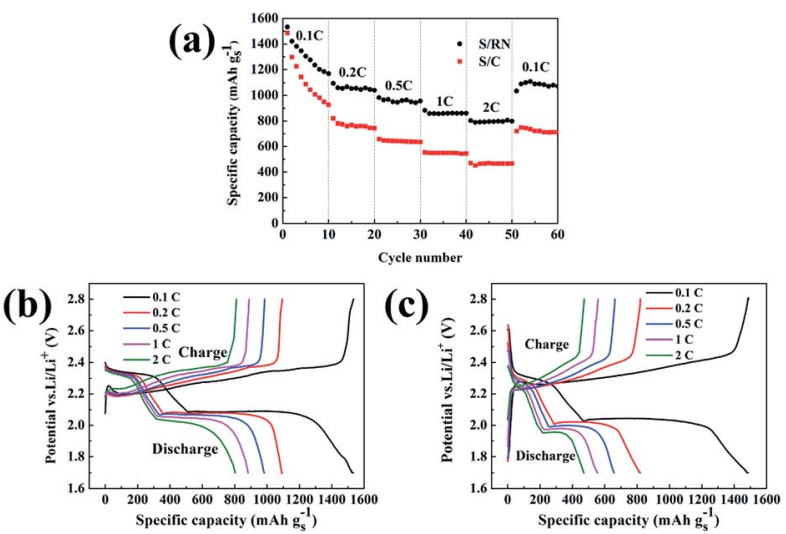

Fig. 7 (a) The discharge capacities of the S/RN and S/C cathodes at the rates of $0.1,0.2,0.5,1$, and $2 \mathrm{C}$, respectively. The galvanostatic 1 st discharge curves at the rates of $0.1,0.2,0.5,1$, and $2 \mathrm{C}$ tested in the range between $1.7-2.8 \mathrm{~V}$ of (b) the S/RN cathode and (c) the S/C cathode. 
To further certify the reaction mechanism of sulfur and RANEY ${ }^{\circledR}$ nickel in the S/RN composite, X-ray photoelectron spectroscopy (XPS) analysis was carried out. Fig. 8a and b show that sulfur loaded in the micropores and mesopores of RANEY® nickel and carbon has different chemical states. The spin-orbit doublet in the $\mathrm{S} / \mathrm{RN}$ composite centered at 164.0 and $162.4 \mathrm{eV}$ is in good agreement with the binding energy of $S 2 p_{1 / 2}$ and $2 p_{3 / 2}$ for $\mathrm{S}_{8}$ molecules. ${ }^{2,10}$ The two satellite peaks at 170.6 and $168.7 \mathrm{eV}$ reveal the existence of smaller, linear $\mathrm{S}_{2-4}$ molecules in the micropores of RANEY® nickel. ${ }^{34}$ The generation of $\mathrm{S}_{2-4}$ molecules may occur in the vacuum process of solvent evaporation. Hence, sulfur can be immobilized in the micropores by physical adsorption on the basis of the porous nature of RANEY ${ }^{\circledR}$ nickel. ${ }^{11}$ The $\mathrm{S} / \mathrm{C}$ composite shows a very weak satellite peak at $169.1 \mathrm{eV}$ without a spin-orbit doublet at 164.4 and $162.8 \mathrm{eV}$. This may be due to $S_{8}$ molecule signals on the surface masking the chain $\mathrm{S}_{2-4}$ in the micropores of carbon, since there are trace amounts of $\mathrm{S}_{2-4}$ molecules. According to previous literature, ${ }^{11,35,36}$ small molecule sulfur of $S_{2-4}$ is more conducive to enhancing the electrochemical performance of $\mathrm{Li}-\mathrm{S}$ batteries as polysulfides can be adsorbed in the micropores of the matrix. The additional peak at $160.1 \mathrm{eV}$ of S $2 \mathrm{p}$ in the S/RN composite demonstrates the formation of $\mathrm{S}^{2-}$. This may suggest the reaction of sulfur and RANEY ${ }^{\circledR}$ nickel, as RANEY ${ }^{\circledR}$ nickel is a highly active material that can even spontaneously burn in the air. ${ }^{37}$ The lower binding energy of $\mathrm{S} 2 \mathrm{p}$ in the $\mathrm{S} / \mathrm{RN}$ composite compared with that of the S/C composite further verifies the chemical adsorption between sulfur and RANEY® nickel. ${ }^{38}$ The electron donation from the elemental nickel improves the electric field, decreasing the energy required for the $\mathrm{S} 2 \mathrm{p}$ electron to be knocked out by the X-rays. ${ }^{10}$ Therefore, the binding energy of $\mathrm{Ni} 2 \mathrm{p}_{3 / 2}$ is higher in the $\mathrm{S} / \mathrm{RN}$ composite than that of RANEY ${ }^{8}$ nickel (Fig. $8 \mathrm{c}$ and d). The reaction between sulfur and nickel can be ascribed to Lewis acid-base binding. ${ }^{39-41}$

The $\mathrm{Ni} 2 \mathrm{p}_{3 / 2}$ spectra of RANEY® nickel and the S/RN composite are depicted in Fig. $8 \mathrm{c}$ and d. The Ni $2 \mathrm{p}_{3 / 2}$ peaks of RANEY® nickel are present at 855.8 and $852.0 \mathrm{eV}$, and can be
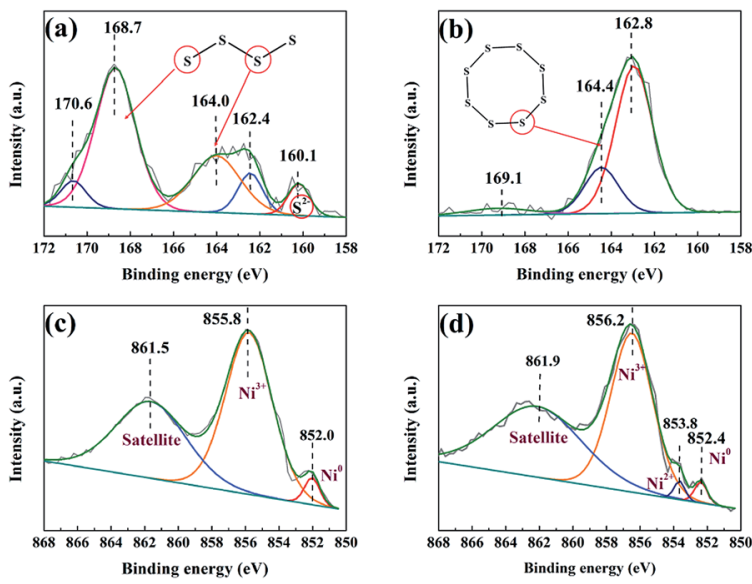

Fig. 8 High-resolution XPS spectra of S $2 p$ for the (a) S/RN and (b) S/C composites before cycling, and of $\mathrm{Ni}_{2} 2 \mathrm{p}_{3 / 2}$ for (c) RN and (d) the S/RN composite before cycling. assigned to the $\mathrm{Ni}^{3+}$ and $\mathrm{Ni}^{0}$ states ${ }^{42,43}$ respectively. The trivalent state of nickel may originate from the interaction of elemental nickel and aluminum during the processes of forming an alloy. There is a spin-orbit doublet at $856.2 \mathrm{eV}$ and a shake-up satellite at $861.9 \mathrm{eV}$ in the $\mathrm{Ni} 2 \mathrm{p}_{3 / 2}$ emission spectrum of the $\mathrm{S} / \mathrm{RN}$ composite. ${ }^{44}$ As shown in Fig. $8 \mathrm{~d}$, an increased peak is located at $853.8 \mathrm{eV}$, representing the $\mathrm{Ni}^{2+}$ species, which further supports the argument that chemical adsorption between sulfur and RANEY® nickel occurs. ${ }^{37}$ The analysis above indicates the existence of the Ni-S chemical bond in the S/RN composite. In addition, according to previous studies, ${ }^{14,16}$ nickel can generate a $\mathrm{Ni}$ chemical bond with polysulfides during the cycling process. Hence, sulfur and polysulfides are both encapsulated in the micropores and mesopores of RANEY ${ }^{\circledR}$ nickel by physical and chemical adsorption, leading to mitigation of the shuttle effect.

From the discussions above, the hypothetical mechanism of immobilizing sulfur grains on the channels of RANEY® nickel is illustrated in Fig. 9. The sulfur grains are infiltrated into the channels of RANEY ${ }^{\circledR}$ nickel by evaporating solvent under vacuum. During this process, $\mathrm{S}_{8}$ and $\mathrm{S}_{2-4}$ molecules are both generated. $\mathrm{S}_{2-4}$ molecules are mostly loaded in the micropores of RANEY ${ }^{\circledR}$ nickel by physical adsorption. ${ }^{35}$ What's more, the RANEY® nickel immobilizer also forms a Ni chemical bond with sulfur grains. During cycling processes, soluble polysulfides can also be restricted by the strong barrier effect of RANEY® nickel acting as a physical and chemical adsorber, and thus the diffusion of polysulfides is suppressed. Furthermore, the decent electrical conductivity of RANEY® nickel also guarantees the elevated performance.

In order to further interpret the reason for the enhanced behavior of the S/RN cathode, EIS measurements were carried out before cycling. As demonstrated in Fig. 10, the Nyquist plots of the S/RN and S/C cathodes have one depressed semicircle at high frequency and an inclined line in the low frequency region. The semicircle in the high frequency region represents the interface charge-transfer resistance $\left(R_{\mathrm{ct}}\right)$. The intercept at the axis $Z^{\prime}$ is in agreement with the combined resistance $\left(R_{\mathrm{e}}\right)$, which is associated with the ionic conductivity of the electrolyte and

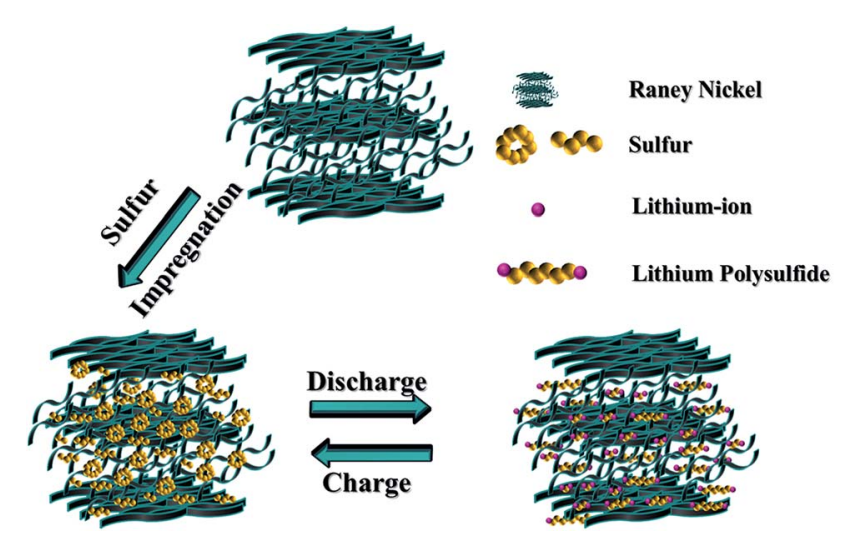

Fig. 9 Schematic demonstration of impregnating sulfur into RANEY ${ }^{\circledR}$ nickel and restricting polysulfides to the channels of RANEY® nickel during cycling. 


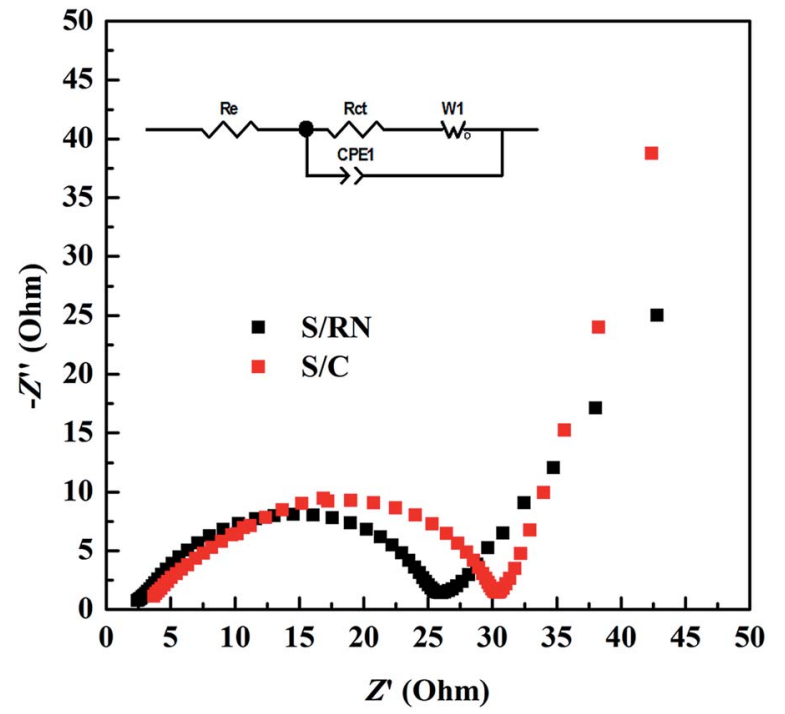

Fig. 10 Nyquist diagrams and the corresponding equivalent circuit of the S/RN and S/C cathodes before discharge/charge cycling.

the contact resistance at the composite/current collector interface. ${ }^{45}$ The $R_{\mathrm{e}}$ value $(2.2 \Omega$ ) of the $\mathrm{S} / \mathrm{RN}$ cathode is lower than that of conventional S/C cathode $(2.4 \Omega)$. This is owing to the firm electrical contact between sulfur and the RANEY ${ }^{\circledR}$ nickel immobilizer due to physical adsorption and the formation of $\mathrm{Ni}$ chemical bonds. In the same way as $R_{\mathrm{e}}$, the $R_{\mathrm{ct}}$ value of the S/RN cathode $(24.3 \Omega)$ is still lower than that of the S/C cathode (28.1 $\Omega$ ) before discharge/charge cycling. The smaller $R_{\mathrm{ct}}$ of the S/RN composite can be ascribed to two reasons. In one respect, sponge-like RANEY® nickel is capable of restraining polysulfides in its channels, which reduces the deposition of insulating $\mathrm{Li}_{2} \mathrm{~S}_{2} / \mathrm{Li}_{2} \mathrm{~S}$ on the cathode surface. Additionally, RANEY® nickel possesses decent electrical conductivity as an alloy. Based on the discussion above, the S/RN cathode exhibits fast charge transfer reaction kinetics resulting in higher cycling performance and rate performance.

\section{Conclusions}

To sum up, we demonstrate the use of RANEY® nickel as a novel immobilizer to encapsulate sulfur, prepared via an accessible solvent and vacuum evaporation induced method, for lithiumsulfur batteries. Sulfur grains are deposited in the micropores and mesopores of RANEY® nickel in the form of $\mathrm{S}_{2-4}$ and $\mathrm{S}_{8}$, respectively. The $\mathrm{S} / \mathrm{RN}$ cathode displays an initial discharge capacity of $1469 \mathrm{~mA} \mathrm{~h} \mathrm{~g}^{-1}$, and achieves a minimal capacity loss of $0.12 \%$ per cycle after 500 cycles with a coulombic efficiency of $99 \%$. The elevated performance of the $\mathrm{S} / \mathrm{RN}$ cathode is ascribed to the physical and chemical coordinated adsorption by RANEY® nickel. The excellent conductivity of RANEY® nickel is conducive to rate capability. Furthermore, the S/RN cathode can also be applied in compact batteries because of its good volumetric specific capacity. It is expected that RANEY® nickel will open a new avenue to high performance and compact $\mathrm{Li}-\mathrm{S}$ batteries.

\section{Acknowledgements}

This study was extraordinarily supported by the National Foundation for Outstanding Basic Research Program of China (2015CB251100).

\section{Notes and references}

1 N.-W. Li, Y.-X. Yin and Y.-G. Guo, RSC Adv., 2016, 6, 617-622.

2 Z. Gong, Q. Wu, F. Wang, X. Li, X. Fan, H. Yang and Z. Luo, RSC Adv., 2016, 6, 37443-37451.

3 M. Raja, N. Angulakshmi and A. M. Stephan, RSC Adv., 2016, 6, 13772-13779.

4 C. Wan, W. Wu, C. Wu, J. Xu and L. Guan, RSC Adv., 2015, 5, 5102-5106.

5 X. Zhou, Q. Liao, T. Bai and J. Yang, J. Mater. Sci., 2017, 52, 7719-7732.

6 X. Li, A. Lushington, Q. Sun, W. Xiao, J. Liu, B. Wang, Y. Ye, K. Nie, Y. Hu, Q. Xiao, R. Li, J. Guo, T. K. Sham and X. Sun, Nano Lett., 2016, 16, 3545.

7 S. Evers, T. Yim and L. F. Nazar, J. Phys. Chem. C, 2012, 116, 19653-19658.

8 S. S. Zhang, J. Power Sources, 2013, 231, 153-162.

9 F. Nitze, K. Fossum, S. Xiong, A. Matic and A. E. C. Palmqvist, J. Power Sources, 2016, 317, 112-119.

10 L. Ma, H. L. L. Zhuang, S. Y. Wei, K. E. Hendrickson, M. S. Kim, G. Cohn, R. G. Hennig and L. A. Archer, ACS Nano, 2016, 10, 1050-1059.

11 S. Rehman, X. Gu, K. Khan, N. Mahmood, W. Yang, X. Huang, S. Guo and Y. Hou, Adv. Energy Mater., 2016, 6, 1502518.

12 J. Zhang, H. Hu, Z. Li and X. W. Lou, Angew. Chem., Int. Ed. Engl., 2016, 55, 3982-3986.

13 Z. Li, C. Li, X. Ge, J. Ma, Z. Zhang, Q. Li, C. Wang and L. Yin, Nano Energy, 2016, 23, 15-26.

14 Q. Zhao, X. Hu, K. Zhang, N. Zhang, Y. Hu and J. Chen, Nano Lett., 2015, 15, 721-726.

15 K. Zhang, F. Qin, J. Fang, Q. Li, M. Jia, Y. Lai, Z. Zhang and J. Li, J. Solid State Electrochem., 2014, 18, 1025-1029.

16 H. Al Salem, G. Babu, C. V. Rao and L. M. Arava, J. Am. Chem. Soc., 2015, 137, 11542-11545.

17 X. Tao, F. Chen, Y. Xia, H. Huang, Y. Gan, X. Chen and W. Zhang, Chem. Commun., 2013, 49, 4513-4515.

18 C. Zhang, W. Lv, Y. Tao and Q.-H. Yang, Energy Environ. Sci., 2015, 8, 1390-1403.

19 X.-B. Cheng, J.-Q. Huang, Q. Zhang, H.-J. Peng, M.-Q. Zhao and F. Wei, Nano Energy, 2014, 4, 65-72.

20 C. Zhang, D. H. Liu, W. Lv, D. W. Wang, W. Wei, G. M. Zhou, S. Wang, F. Li, B. H. Li, F. Kang and Q. H. Yang, Nanoscale, 2015, 7, 5592-5597.

21 X. Wang, T. Gao, F. Han, Z. Ma, Z. Zhang, J. Li and C. Wang, Nano Energy, 2016, 30, 700-708.

22 S. Taccola, F. Greco, E. Sinibaldi, A. Mondini, B. Mazzolai and V. Mattoli, Adv. Mater., 2015, 27, 1668-1675.

23 X. Wang, Z. Zhang, Y. Qu, Y. Lai and J. Li, J. Power Sources, 2014, 256, 361-368.

24 P. Fouilloux, Appl. Catal., 1983, 8, 1-42. 
25 J. Freel, W. J. M. Pieters and R. B. Anderson, J. Catal., 1969, 14, 247-256.

26 J. J. Cheng, J. T. Zhu, Y. Pan, Z. S. Ma, H. J. Song, J. A. Pan, Z. Z. Li and C. Lu, ECS Electrochem. Lett., 2014, 4, A19-A21.

27 X. Zhou, Q. Liao, J. Tang, T. Bai, F. Chen and J. Yang, J. Electroanal. Chem., 2016, 768, 55-61.

28 K. Ding, Q. Liu, Y. Bu, K. Meng, W. Wang, D. Yuan and Y. Wang, J. Alloys Compd., 2016, 657, 626-630.

29 P. Wei, M. Q. Fan, H. C. Chen, X. R. Yang, H. M. Wu, J. Chen, T. Li, L. W. Zeng, C. M. Li, Q. J. Ju, D. Chen, G. L. Tian and C. J. Lv, Renewable Energy, 2016, 86, 148-153.

30 Q. Qu, T. Gao, H. Zheng, Y. Wang, X. Li, X. Li, J. Chen, Y. Han, J. Shao and H. Zheng, Adv. Mater. Interfaces, 2015, 2, 1500048.

31 J.-H. Wang and M. Liu, Electrochem. Commun., 2007, 9, 22122217.

32 X. Qian, L. Jin, D. Zhao, X. Yang, S. Wang, X. Shen, D. Rao, S. Yao, Y. Zhou and X. Xi, Electrochim. Acta, 2016, 192, 346-356.

33 C. Y. Fan, P. Xiao, H. H. Li, H. F. Wang, L. L. Zhang, H. Z. Sun, X. L. Wu, H. M. Xie and J. P. Zhang, ACS Appl. Mater. Interfaces, 2015, 7, 27959-27967.

34 Y. Tao, Y. Wei, Y. Liu, J. Wang, W. Qiao, L. Ling and D. Long, Energy Environ. Sci., 2016, 9, 3230-3239.
35 S. Xin, L. Gu, N. H. Zhao, Y. X. Yin, L. J. Zhou, Y. G. Guo and L. J. Wan, J. Am. Chem. Soc., 2012, 134, 18510-18513.

36 Z. Zhang, Z. Li, F. Hao, X. Wang, Q. Li, Y. Qi, R. Fan and L. Yin, Adv. Funct. Mater., 2014, 24, 2500-2509.

37 L.-J. Liu, Y. Chen, Z.-F. Zhang, X.-L. You, M. D. Walle, Y.-J. Li and Y.-N. Liu, J. Power Sources, 2016, 325, 301-305.

38 K. Mi, Y. Jiang, J. Feng, Y. Qian and S. Xiong, Adv. Funct. Mater., 2016, 26, 1571-1579.

39 X. Wang, T. Gao, X. Fan, F. Han, Y. Wu, Z. Zhang, J. Li and C. Wang, Adv. Funct. Mater., 2016, 26, 7164-7169.

40 X. Liang, A. Garsuch and L. F. Nazar, Angew. Chem., 2015, 54, 3907-3911.

41 J. Zheng, J. Tian, D. Wu, M. Gu, W. Xu, C. Wang, F. Gao, M. H. Engelhard, J. G. Zhang and J. Liu, Nano Lett., 2014, 14, 2345.

42 A. A. AbdelHamid, X. Yang, J. Yang, X. Chen and J. Y. Ying, Nano Energy, 2016, 26, 425-437.

43 Q. Li, J. Ma, H. Wang, X. Yang, R. Yuan and Y. Chai, Electrochim. Acta, 2016, 213, 201-206.

44 L. Niu, Y. Wang, F. Ruan, C. Shen, S. Shan, M. Xu, Z. Sun, C. Li, X. Liu and Y. Gong, J. Mater. Chem. A, 2016, 4, 56695677.

45 X. Liu, K. Zhu, J. Tian, Q. Tang and Z. Shan, J. Solid State Electrochem., 2014, 18, 2077-2085. 